\title{
GRANDE-BRETAGNE
}

\author{
PRÉSIDENCE DE LA SOCIÉTÉ
}

La mort de Lord Wantage ${ }^{1}$ avait laissé vacant la place de président de la Société anglaise de secours aux militaires blessés et malades. Lord Rothschild, qui était membre du Conseil depuis 1870, a été appelé à l'unanimité à revêtir ces fonctions.

\section{LORD WANTAGE}

(Nécrologie).

Nous avons annoncé dans notre précédent Bulletin ${ }^{1}$ la mort du président de la Société anglaise de secour's aux malades et blessés en cas de guerre (Croix-Rouge anglaise). Sur notre demande, la Société a bien voulu nous communiquer sur son compte les détails biographiques suivants:

Robert-James Loyd-Lindsay, baron Wantage, avait fait la guerre de Crimée et s'y était acquis une grande réputation de bravoure; aux bataille d'Almas et d'Inkermann il gagıa la croix Victoria. Dès le début de sa carrière militaire, il se fit connaître également par ses efforts en faveur des soldats anglais en général, et plus particulièrement par sa profonde sympathie pour les malades et les blessés des champs de bataille.

Dès 1870 il s'occupa en Angleterre de l'œuvre de la Croix-Rouge, prit la haute main dans la fondation et l'organisation de la Société anglaise, laquelle, sous sa direction et celle de ses collègues, est depuis généreusement intervenue dans toutes les guerres, pour soulager les blessés et les malades.

Au début de la guerre franco-allemande, le public anglais, sur les sollicitations et les efforts de Lord Wantage, souscrivit une

1 Voy. p. 166. 
somme de $£ 300,000$, qui permit d'envoyer d'importants secours sur les champs de bataille. Non content de fonctionner comme président de la Société, Lord Wantage visita personnellement le. théâtre des opérations et versa de sa poche une contribution de $£ 40,000$, en faveur des blessés français aussi bien qu'allemands qni se trouvaient à Paris et à Versailles. Le roi de Prusse lui accorda la permission d'entrer dans Paris le 11 octobre 1870, et son action charitable en faveur des victimes en devint plus efficace encore. Le roi lui exprima personnellement sa reconnaissance pour son magnifique don, fait précisément à une époque où les ressources et les soins faisaient défaut.

De même, pendant la guerre turco-serbe en 1876 , il surveilla luimême les opérations de la Société sur le théâtre de la guerre, établissant des hôpitaux et les pourvoyant de matériel.

La sage administration des finances de la Société en temps de paix lui permettait ainsi de consacrer d'importantes sommes à réaliser le but social en temps de guerre. Cette ligne de conduite, bien que n'étant pas en harmonie avec celle que suivaient les sociétés du continent, rendit inutile tout appel au public jusqu'à la guerre du Transvaal.

L'œuvre de la Société au sud de l'Afrique n'est pas encore terminée et un compte rendu complet ne peut pas encore en être donné. Jusqu'ici une somme de $£$ 180,000 (4,500,000 francs) a été, récoltée et presque entièrement dépensée à affrêter un navire hôpital, à organiser et à entretenir un train hôpital, à équiper des diaconesses (nurses) et des brancardiers, enfin à distribuer libéralement des vêtements et des cordiaux aux malades et aux blessés.

Lord Wantage fut appelé également à la présidence du Comité central anglais, lors de la fondation de ce Comité, créé peu de temps avant l'ouverture des hostilités sud-africaines ${ }^{1}$, en vue d'organiser et de centraliser toute l'assistance volontaire.

La direction de la Société anglaise faisait reposer sur Lord Wantage une lourde responsabilité, et malgré sa santé ébranlée, il continua à se dépenser pour elle jusqu'à la veille de sa mort.

La perte que celle-ci fait en lui est considérable, son talent d'organisation et son enthousiasme généreux pour la cause qu'elle

1 Voy. T XXXI, p. 29. 
soutient lui manqueront cruellement. Puisse cependant son labeur de plus de trente années lui survivre et la Société continuer à jouir de la prospérité qu'il a su lui donner.

\section{MECKLEMBOURG}

\section{LA SOCIÉTÉ MECKLEMBOURGEOISE DE 1898 a 1900}

La Société mecklembourgeoise, qui ne publie, comme on sait, que tous les deux ans un rapport sur son activité, ne tient que tous les trois ans son assemblée générale. Elle n'en a donc pas eu de 1898 à 1900. Le changement de nom de la Société a été ratifié par le duc régent; elle a donc été définitivement baptisée «Société mecklembourgeoise de la Croix-Rouge .

Le président von Schuckmann a donné sa démission, pour cause de surcroît d'occupations; il a été remplacé par le secrétaire von Hirschfeld.

L'organisation intérieure de la Société a fait un pas en avant, par la création d'une section locale à Grasse, laquelle pourrait déjà mettre en ligne, en temps de guerre, un lazaret de 15 lits.

Au 30 juin 1900, la Société possédait 15 comités locaux. Elle a, en outre, enfin acquis une demeure qui lui est propre. Cette maison, entourée d'un jardin et située tout près de la gare, présente tous les avantages possibles. Pour lui permettre l'acquisition de cet immeuble, le Comité cen tral allemand lui a faitl'avance d'une somme de $30,000 \mathrm{Mk}$. remboursable en trois ans.

En vue de développer les colonnes sanitaires, le Comité n'a pas négligé d'envoyer à l'assemblée des médecins et des chefs de colonnes des représentants qui en ont rapporté des enseignements féconds.

Un exercice général de toutes les colonnes sanitaires mecklembourgeoises a eu lieu le 9 octobre 1898 sur la place de Schwerin; $\mathrm{y}$ ont pris part 15 médecins, 30 chefs, et 249 porteurs avec 62 civières.

Sur l'instigation du Comité central allemand, la Société a parti- 